\title{
High-Resolution Contact Printing with Chemically Patterned Flat Stamps Fabricated by Nanoimprint Lithography
}

\author{
By Xuexin Duan, Yiping Zhao, András Perl, Erwin Berenschot, David \\ N. Reinhoudt, and Jurriaan Huskens*
}

Microcontact printing $(\mu \mathrm{CP})$ has developed rapidly into a robust printing tool over the past few years because of its processing advantages in comparison to conventional lithographic techniques. ${ }^{[1-4]}$ In a typical $\mu \mathrm{CP}$ scheme, a soft elastomeric stamp is brought into intimate contact with a substrate to transfer ink molecules from the stamp to the substrate. Contact printing is not diffraction limited, and can in principle be used to pattern surfaces with sub-100 nm features. However, deformation of the soft-polymer stamps due to the limited mechanical stability of the elastomer during stamp fabrication or printing, leading to pairing, buckling, or roof collapse of structures, renders them unsuitable for high-resolution $\mu \mathrm{CP} .{ }^{[5-8]}$ New stamp materials ${ }^{\left[{ }^{[9-13]}\right.}$ and composite stamps ${ }^{[6,14,15]}$ may overcome these problems.

The mechanical issues are a direct consequence of the inclusion of voids as the transport barriers in topographically patterned stamps. Recently, a new transport-barrier concept was introduced using chemical patterns on a flat stamp surface as the barriers to transfer ink molecules either from the chemically patterned areas ${ }^{[16]}$ or from the areas in between. ${ }^{[17,18]}$ In principle, using a chemically patterned flat stamp can solve many or all stamp-stability issues.

A typical way to create chemical patterns on a flat stamp surface is the oxidation of a flat PDMS surface in an oxygen plasma using a shadow mask, followed by stabilization of the hydrophilized areas by reaction with silanes. Functionalization by $1 H, 1 H$, $2 \mathrm{H}, 2 \mathrm{H}$-perfluorodecyl-trichlorosilane (PFDTS) rendered it possible to transfer regular thiol inks using such flat stamps. ${ }^{[18]}$ However, the feature size is limited by the shadow mask used. The smallest commercially available shadow mask has $350 \mathrm{~nm}$ features. Another limitation is the difficulty to maintain conformal contact between the mask and the stamp surface during the stamp-oxidation step when a sub-500 nm mask is used, thus leading to loss of resolution. Here, we report a new

[*] Prof. J. Huskens, X. Duan, Y. Zhao, Dr. A. Perl, Prof. D. N. Reinhoudt Molecular Nanofabrication Group

$\mathrm{MESA}+$ Institute for Nanotechnology

University of Twente

P. O. Box 217, 7500 AE Enschede (The Netherlands)

E-mail: j.huskens@utwente.nl

Y. Zhao, E. Berenschot

Transducers Science and Technology Group

MESA+ Institute for Nanotechnology

University of Twente

P. O. Box 217, 7500 AE Enschede (The Netherlands)

DOI: $10.1002 / \mathrm{adma.200803809}$ method to prepare chemically patterned flat PDMS stamps using nanoimprint lithography (NIL). Printing of alkanethiols on gold is shown to result in sub-100 $\mathrm{nm}$ contact printing.

NIL has been demonstrated to be a high-volume and cost-effective patterning technique with sub-10 $\mathrm{nm}$ resolution. ${ }^{[19]}$ In this process, a hard mold with nanoscale features is pressed into a thin polymer film that has been coated on a substrate, deforming the shape of the polymer according to the features of the mold and forming a relief pattern in the polymer layer. The patterned polymer layer can be further processed by reactive ion etching, for instance, to transfer the pattern onto the underlying substrate. Here, we used the NIL-patterned polymer film on a flat PDMS substrate as a mask in the oxidation step to fabricate the chemically patterned flat stamp (Scheme 1).

The polymer pattern, produced by NIL followed by residuallayer removal, was used as a local mask to oxidize the flat PDMS substrate on the uncovered regions. Subsequently, the chemical patterns were formed by gas-phase evaporation of the fluorinated silane. In the last step, the residual polymer was removed in a solvent.

Thermal NIL is based on the mechanical molding of a thermoplastic polymer normally on a hard substrate. However, when using PDMS as an imprint substrate, the poor mechanical properties of the PDMS may lead to pattern distortions during the thermal imprint process. Therefore, we fabricated a composite flat PDMS substrate consisting of commercial PDMS (Sylgard 184) with a $150 \mu \mathrm{m}$ cover glass as a support layer (see Supporting

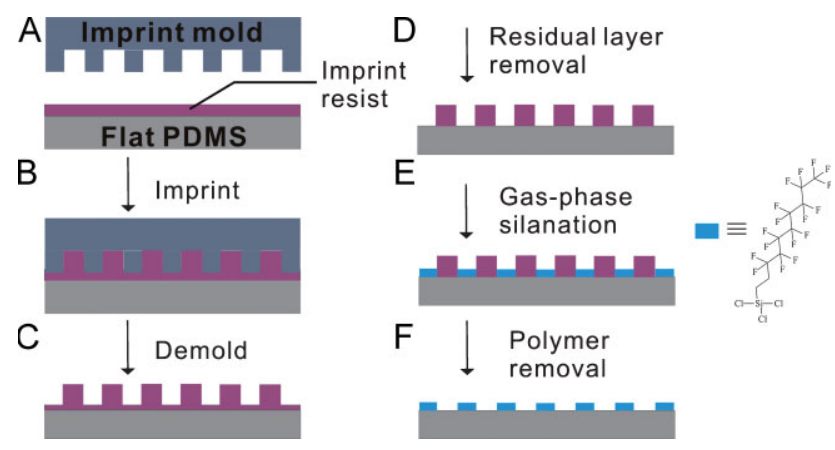

Scheme 1. Schematic illustration of the stamp fabrication: a) Spin coating of imprint resist mr-I T85 and prebaking. b) Imprinting the mr-I T85 layer using the NIL mold. c) Demolding of the mold from the PDMS substrate. d) $\mathrm{O}_{2}$ RIE for the "window opening" down to the PDMS substrate and oxidation of the PDMS. e) Gas-phase silanation of PFDTS onto the uncovered PDMS. (f) Lift-off of the residual mr-I T85 in cyclohexane. 
Information). The advantage of this method is that the thin glass gives the stamp enough support during imprint, while the composite stamp has enough flexibility to achieve good contact in the contact-printing step.

Another important requirement in the NIL process is the selection of a suitable polymeric resist material. We have used mr-I T85 as the imprint resist, a thermoplastic polymer with a $T_{\mathrm{g}}$ of $85^{\circ} \mathrm{C}$ that can be imprinted at a relatively low temperature $\left(130-150^{\circ} \mathrm{C}\right)$. Because of the more apolar nature of $\mathrm{mr}$-I T85, the adhesion to the hydrophobic PDMS substrate is better compared to the more commonly used PMMA.

Before spin-coating of the imprint resist, the composite PDMS substrate was treated shortly with $\mathrm{O}_{2}$ plasma to achieve a better film quality. The spin-coated-film thickness was calculated according to the different pattern sizes and pattern density. Fabrication of NIL patterns with a minimal residual layer is essential to obtain well-defined nanometer-sized patterns, because prolonged exposure to oxygen plasma, which is used in the next step to remove the residual layer, can lead to physical damage of the PDMS surface producing "cracks" or other microstructures. ${ }^{[20]}$ In order to avoid damage to the PDMS surface and to have a more anisotropic etching, the amount of the applied imprint resist was tuned so that the thickness of the residual layer was less than $10 \mathrm{~nm}$. a

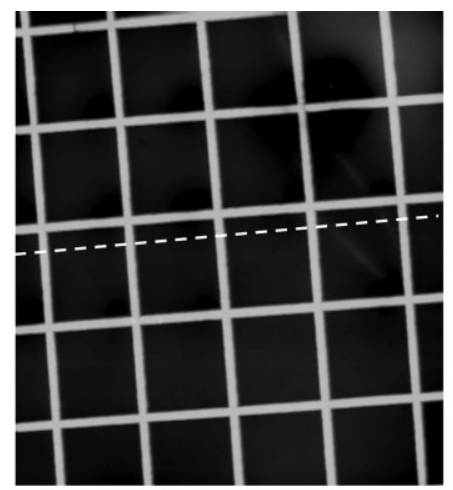

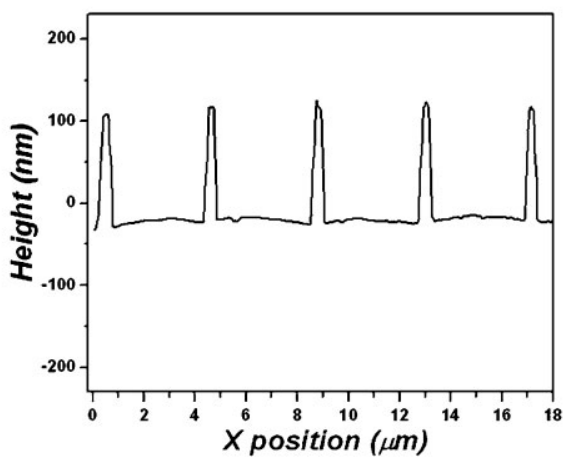

b

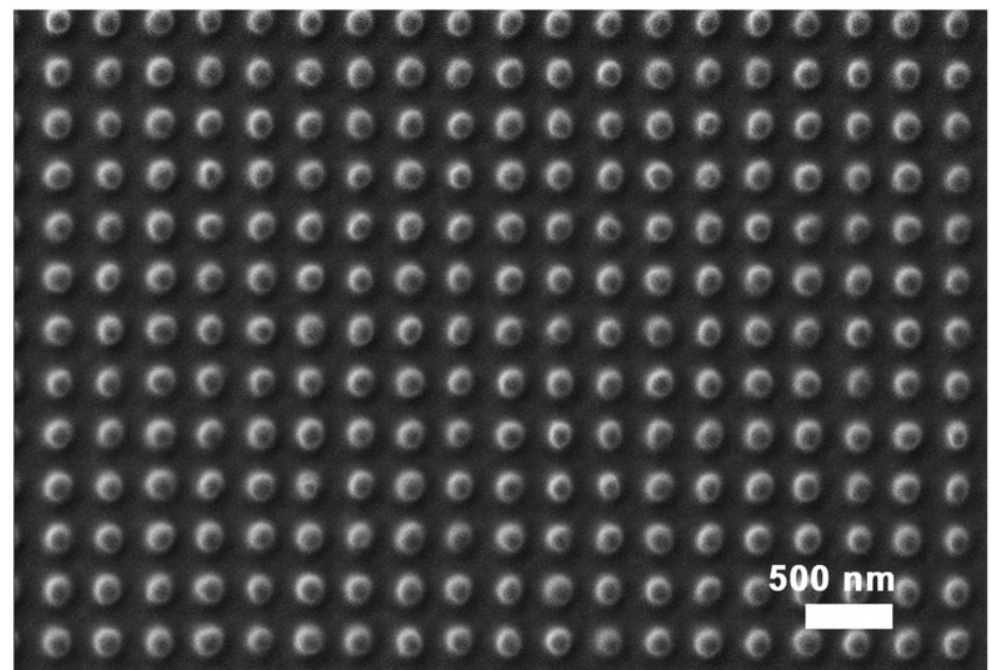

Figure 1. a) AFM height image $(20 \mu \mathrm{m} \times 20 \mu \mathrm{m})$ and its corresponding cross-sectional profile and b) SEM image of the imprinted (mr-I T85) patterns on composite PDMS substrates.
Initially, two kinds of templates were used, one with a $500 \mathrm{~nm}$ cross bar with a $4 \mu \mathrm{m}$ period (depth $150 \mathrm{~nm}$ ), and another containing holes with half pitch down to $150 \mathrm{~nm}$ (depth $100 \mathrm{~nm}$ ). Before NIL, the molds were coated with PFDTS vapor, as an antiadhesion layer for better mold release. ${ }^{[21]}$ Figure 1 shows the imprinted polymer patterns with the two different molds on PDMS substrates. From the atomic force microscopy (AFM) cross-section (Fig. 1a) it is clear that the polymers have filled all cavities of the mold. In case of dot patterns, replication was also faithful judging from the feature sizes $((150 \pm 5) \mathrm{nm})$, which are identical to the original mold features.

An important observation was that, when using PDMS as the imprint substrate, NIL can be performed at very low pressures. A pressure of only $2 \mathrm{bar}\left(1 \mathrm{bar}=10^{5} \mathrm{~Pa}\right.$ ) was sufficient to achieve a good replica during the imprint process. It has been reported before that the imprint pressure can be significantly reduced if a flexible mold is used, which is attributed to conformal contact. ${ }^{[2]}$ The same phenomenon is observed here when using a flexible PDMS substrate. Such a low-pressure process is an advantage in preventing damage to the mold and distortion of the composite PDMS substrate during imprint.

Residual-layer removal and activation of the exposed areas of the PDMS were achieved in a single $\mathrm{O}_{2}$ reactive-ion-etching (RIE) step. Since the residual polymer layer is $\sim 10 \mathrm{~nm}$, the oxygen-plasma treatment can be relatively short, thus minimizing damage of the imprinted patterns during etching. Figure 2 shows AFM and scanning electron microscopy (SEM) images of the patterns after residuallayer removal by $\mathrm{O}_{2}$ RIE. The decrease of the polymer height $(30 \mathrm{~nm})$ indicates the successful removal of the residual layer. Measured from the SEM image, the diameter of the dots patterns is $(140 \pm 10) \mathrm{nm}$, confirming that RIE did not significantly modify the lateral dimensions of the imprinted features.

Immediately after $\mathrm{O}_{2}$ RIE, PFDTS was deposited from the vapor phase onto the locally oxidized PDMS substrates. The adsorbed PFDTS is assumed to react with the silanol groups present on the oxidized PDMS to yield a PFDTS-modified PDMS stamp. ${ }^{[18]}$

Subsequently, the remaining polymer resist was selectively removed by dipping the PDMS substrates into hot cyclohexane $\left(80^{\circ} \mathrm{C}\right)$ for 1 min. It is known that PDMS swells in contact with nonpolar solvents (such as hydrocarbons, toluene, and dichloromethane). ${ }^{[23]}$ The nonpolar cyclohexane causes some swelling of the PDMS stamp after $1 \mathrm{~min}$, but this effect was completely reversible upon applying a vacuum. The swelling did not influence the chemical patterns on the stamp surface, as proven by the $\mu \mathrm{CP}$ results shown below.

The PFDTS-modified flat PDMS stamps were used to transfer patterns of octadecanethiol (ODT) onto gold substrates by $\mu \mathrm{CP}$. After printing, the gold substrates were 

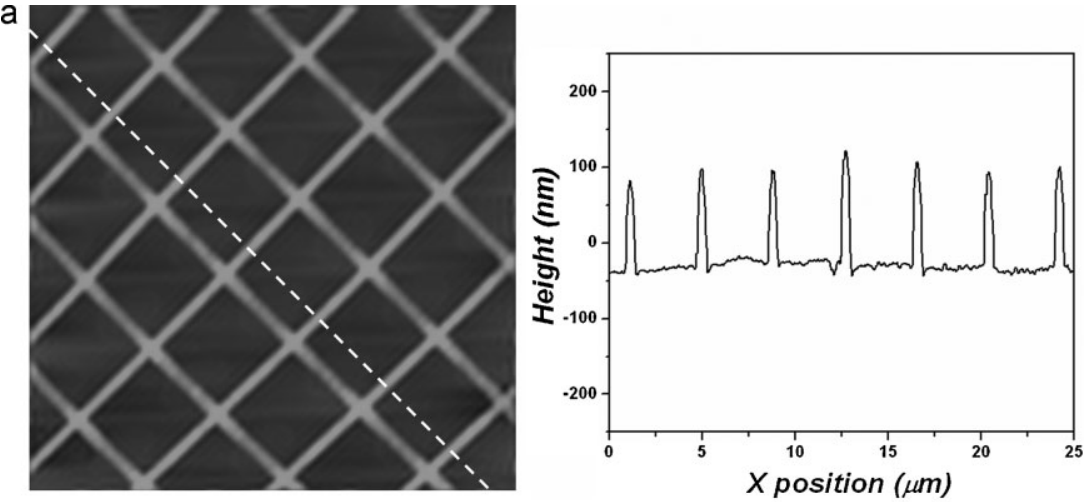

b

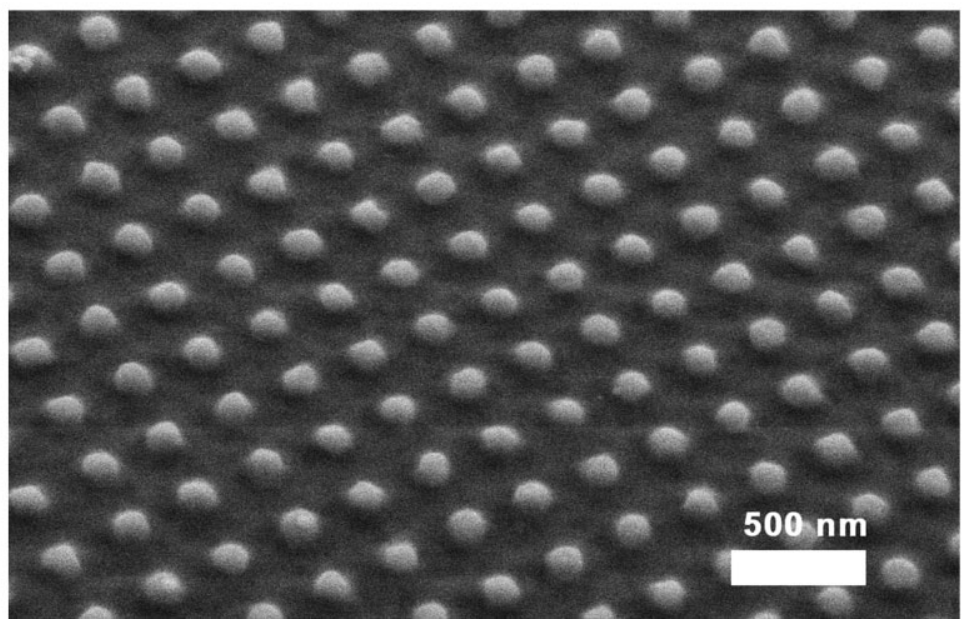

Figure 2. a) AFM height image $(20 \mu \mathrm{m} \times 20 \mu \mathrm{m})$ and its corresponding cross-sectional profile and b) SEM image of the mr-I T85 patterns after $\mathrm{O}_{2}$ RIE.

selectively etched by a ferri-ferrocyanide-based etching solution. ${ }^{[2]}$ Figure 3 shows AFM and SEM images of gold patterns generated by $\mu \mathrm{CP}$ using PFDTS-modified flat PDMS stamps and subsequently wet etching.

As seen from the AFM height image and height profile (Fig. 3a), the gold patterns have a uniform depth of $20 \mathrm{~nm}$. The width of the cross bar lines closely matches the original imprint mold. In case of the dot patterns (Fig. 3b), the gold dots have a diameter of $(130 \pm 15) \mathrm{nm}$. The slight decrease $(10 \mathrm{~nm}$ per side) compared to the original mold is attributed to the isotropic wet etching of the gold. ${ }^{[24]}$

To test the limitations of the PFDTS-modified flat PDMS stamps, sub- $100 \mathrm{~nm}$ molds were used to fabricate chemical patterns on the PDMS surface. A mold with nanoridges $(50 \mathrm{~nm}$ wide, $100 \mathrm{~nm}$ high, separated by $4 \mu \mathrm{m}$ ) was fabricated by edge lithography followed by wet etching (Fig. 4a). ${ }^{[25]}$ Figure $4 \mathrm{~b}$ shows the polymer patterns after the imprint on the PDMS substrate. Polymer nanogrooves of $(50 \pm 5) \mathrm{nm}$ separated by $4 \mu \mathrm{m}$ were formed on the PDMS surface. After the polymer patterns were converted into chemical patterns, the PFDTS-modified flat PDMS stamps were used in $\mu \mathrm{CP}$. Both regular $(-) \mu \mathrm{CP}$ and positive printing $((+) \mu \mathrm{CP})$ were used to produce gold nanopatterns upon wet etching. Upon $(-) \mu \mathrm{CP}$ using ODT, gold nanogrooves were formed with a width of $(95 \pm 25) \mathrm{nm}$ (Fig. 4c). The widening is again attributed to the wet-etching step. Upon $(+) \mu \mathrm{CP}$ using a thioether-modified poly(propylene imine) dendrimer, ${ }^{[26]}$ high-quality nanolines were formed after gold etching (Fig. 4d) with an average size of $(85 \pm 10) \mathrm{nm}$. The slight increase of the width of the gold lines, which is again fully attributable to the wet-etching step, also indicates that ink diffusion is not an issue when printing with such a flat stamp. Thus, in principle, even smaller features can be fabricated when using a high-resolution mold.

PFDTS, when attached to oxidized PDMS, is reported to have a surface free energy as low as $12.7 \times 10^{-3} \mathrm{~J} \mathrm{~m}^{-2}$.[27] Therefore, PFDTS-modified chemically patterned PDMS stamps are quite stable. ${ }^{[18]}$ We have used the same stamp to print 10 times, over a period of one month. The quality of the etched substrates was identical and independent of the age of the stamp.

In summary, nanoimprint lithography can be combined with microcontact printing to achieve high-resolution printing. Thermal NIL can be used to fabricate polymer patterns on flat PDMS substrates at low pressures. The polymer pattern can be used as a local mask to oxidize the flat PDMS substrate at the uncovered regions, in order to produce flat, chemically patterned stamps. Sub- $100 \mathrm{~nm}$ gold lines were successfully replicated by these stamps. The resolution is limited only by the mold used in the NIL step and by the wet-etching step (when metal patterns are targeted). The combined NIL- $\mu \mathrm{CP}$ procedure embodies advantages of NIL (high resolution) with those of $\mu \mathrm{CP}$, both regarding the flexible nature of PDMS (conformal contact in both the NIL and printing steps) and the flat stamp concept (high resolution, elimination of ink diffusion and stamp deformation). It thus provides a versatile option to create high-resolution patterns, especially when combined with the large chemical versatility of $\mu \mathrm{CP}$.

\section{Experimental}

Materials and Methods: PFDTS (purity 97\%), was purchased from ABCR GmbH. Imprint resist mr-I T85 was obtained from Micro Resist. All materials were used as received without further purification. The third generation of thioether-modified poly(propylene imine) dendrimers (G3-M) was synthesized according to a reported procedure [26].

Composite Flat PDMS Substrates: Composite flat PDMS substrates were prepared by first casting the liquid prepolymer of the elastomer onto a polished $\mathrm{Si}$ wafer onto which an antiadhesion layer had been deposited. After degassing, a piece of cover glass (MENZEL-GLÄSER BB015015A1 $15 \mathrm{~mm} \times 15 \mathrm{~mm}$ ) that has been cleaned using piranha solution (3:1 mixture of sulfuric acid and hydrogen peroxide. CAUTION! Piranha solutions should be handled with great care in open containers in a fume hood. Piranha is highly corrosive and toxic and potentially explosive) was then put into the uncured mixture. The cover glass sank to the bottom of the PDMS liquid leaving a thin layer between the cover glass and the Si wafer. After curing at $60^{\circ} \mathrm{C}$ for $8 \mathrm{~h}$, the stamp was peeled off from the Si wafer. 

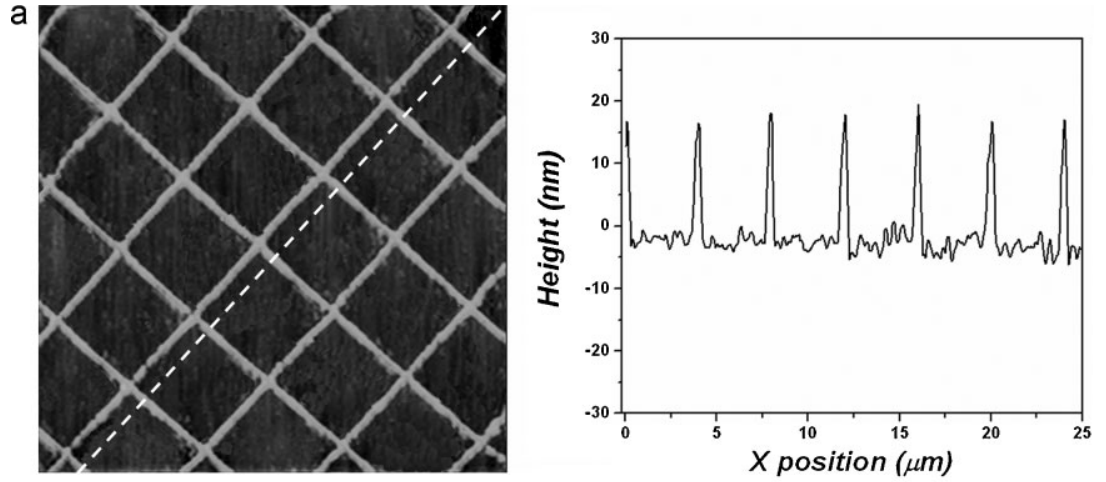

b

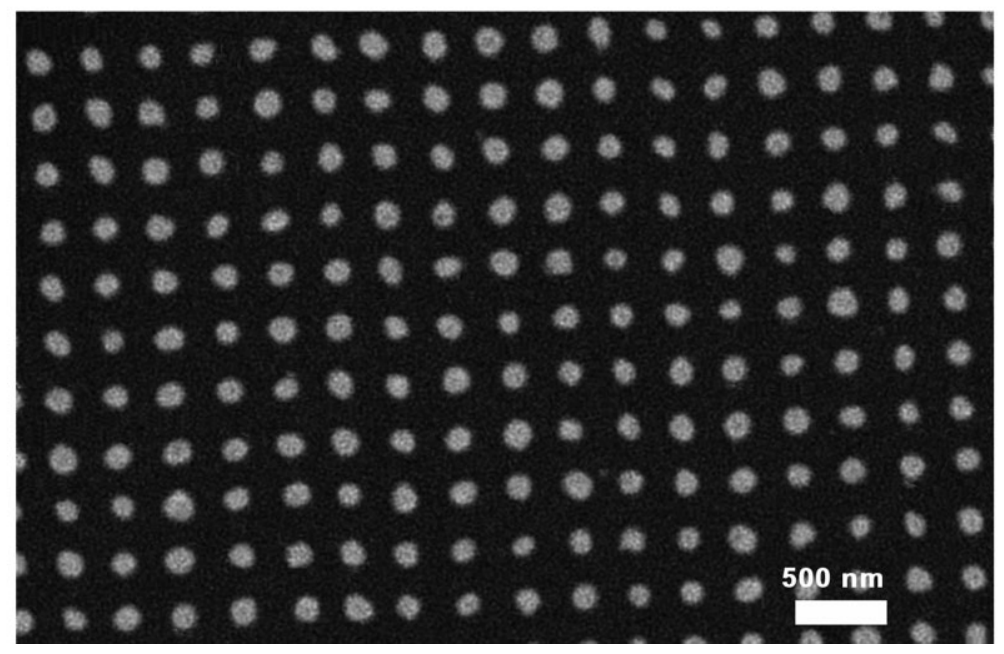

Figure 3. a) AFM height image $(20 \mu \mathrm{m} \times 20 \mu \mathrm{m})$ and its corresponding cross-sectional profile and b) SEM image of the etched gold, which were patterned using ODT transferred from PFDTS-modified patterned flat PDMS stamps (1 mM ODT; printing time: 1 min; etch time: 7 min).

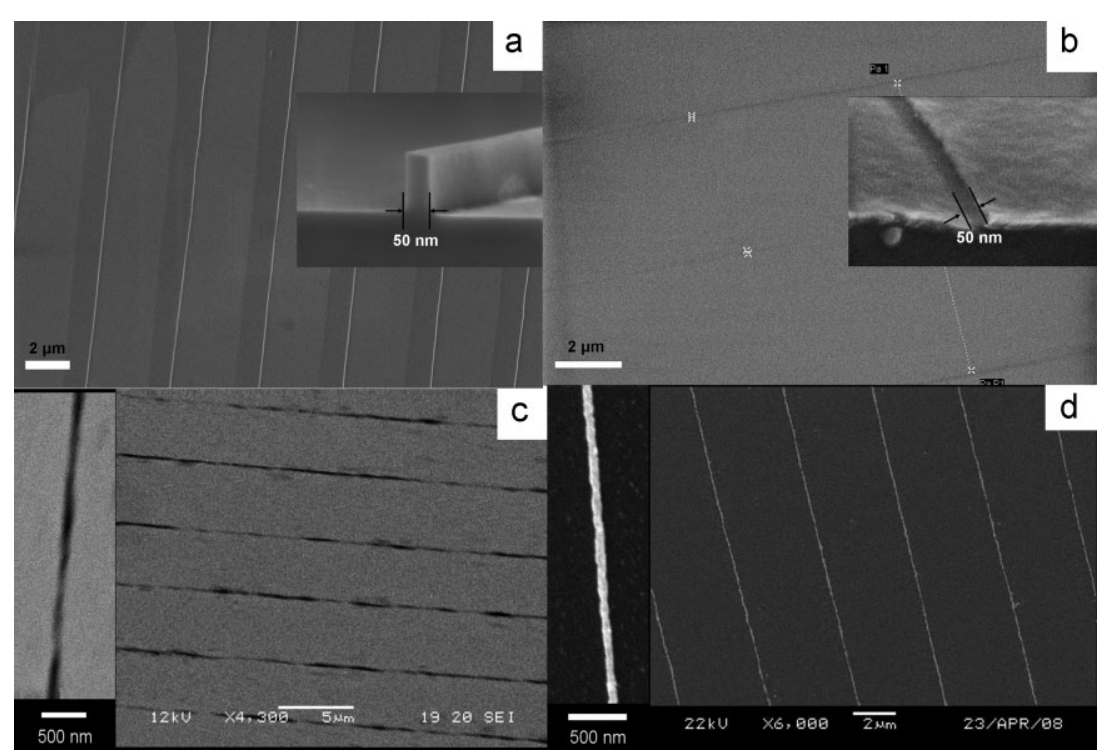

Figure 4. a) SEM images of sub- $100 \mathrm{~nm}$ mold, b) the imprinted ( $\mathrm{mr}-\mathrm{I}$ T85) patterns on composite PDMS substrate. Etched gold patterned using c) $(-) \mu \mathrm{CP}$ and d) $(+) \mu \mathrm{CP}$ using a sub- $100 \mathrm{~nm}$ PFDTS-modified patterned flat PDMS stamp.
NIL Procedure: Before spin-coating the resist, the composite PDMS substrate was treated by short $\mathrm{O}_{2}$ plasma ( $10 \mathrm{mTorr} ; 10 \mathrm{~W} ; 20 \mathrm{sccm} \mathrm{O} ; 15 \mathrm{~s}$ ). This oxidation step increases the surface energy of the PDMS substrate to achieve a better film quality. A thin film of mr-I T85 solution was spin-coated onto the substrates, followed by a soft baking step for $2 \mathrm{~min}$ at $140{ }^{\circ} \mathrm{C}$ on a hot plate.

The imprint templates were purchased from NIL Technology, one with a $500 \mathrm{~nm}$ cross bar with a $4 \mu \mathrm{m}$ period (depth $150 \mathrm{~nm}$ ), and another containing holes with half pitch down to $150 \mathrm{~nm}$ (depth $100 \mathrm{~nm}$ ). PFDTS was used as an antiadherent layer to facilitate the stamp-imprint separation.

A stack of a composite PDMS substrate coated with mr-l T85 and a Si template were inserted into a hydraulic press (Specac), and the temperature was raised to $140{ }^{\circ} \mathrm{C}$. Then, 2 bar of pressure was applied to the system for $5 \mathrm{~min}$. Upon cooling to $50^{\circ} \mathrm{C}$, the template was separated from the PDMS substrate.

Pattern Transfer: After imprinting, residual-layer removal down to the PDMS substrate was achieved via $\mathrm{O}_{2}$ RIE ( $10 \mathrm{mTorr}, 10 \mathrm{~W}, 20 \mathrm{sccm} \mathrm{O}$,). The etch rate of T85 varied from 1 to $5 \mathrm{~nm} \mathrm{~s}^{-1}$, depending on the pattern sizes and pattern density. In order to activate the exposed PDMS after residual-layer removal, the $\mathrm{O}_{2}$-plasma treatment was extended for another $15 \mathrm{~s}$.

Directly after $\mathrm{O}_{2}$ RIE, PFDTS was immediately deposited for $30 \mathrm{~min}$ from vapor phase onto the locally oxidized PDMS substrates. The adsorbed PFDTS was assumed to react with the silanol groups present on the oxidized PDMS to yield a PFDTS-modified chemically patterned PDMS stamp [18]. The substrates were thereafter cured in an oven for $3 \mathrm{~h}$ at $60^{\circ} \mathrm{C}$ to complete the reaction.

Contact Printing: Gold substrates were obtained from Ssens BV (Hengelo, The Netherlands) as a layer of $20 \mathrm{~nm}$ gold on titanium $(2 \mathrm{~nm})$ on silicon. Before use, the substrates were treated with oxygen plasma, immersed in ethanol for $1 \mathrm{~h}$, rinsed with water (Millipore) and ethanol, and dried with nitrogen.

The stamps were inked with a few drops of ODT solution in ethanol $(1 \mathrm{~mm})$ and dried in a stream of nitrogen. After drying with nitrogen, the stamps were placed manually on the substrate, and were pressed slightly against the gold surface at the initial stage of the printing to induce conformal contact. The printing time was $1 \mathrm{~min}$ and the gold samples were selectively etched by keeping the substrate in $\mathrm{S}_{2} \mathrm{O}_{3}{ }^{2-}$ / ferri/ferrocyanide-based etching solutions for different times (in case of features $>100 \mathrm{~nm}, 7$ min etching is used; in the case of sub- $100 \mathrm{~nm}$ nanogrooves, $9 \mathrm{~min}$ etching is needed to get a clear contrast).

In $(+) \mu \mathrm{CP}$ of the third generation poly (propylene imine) dendrimer with dialkyl sulfide end groups (G3-M) [26], the stamp was inked with a solution of G3-M $\left(4 \times 10^{-2} \mathrm{~mm}\right)$. Printing was performed for $2 \mathrm{~min}$, and the substrate was subsequently dipped in a $0.1 \mathrm{~mm}$ ODT solution for $5 \mathrm{~s}$. A freshly prepared etching solution consisting of $10 \mathrm{mM} \mathrm{Fe}\left(\mathrm{NO}_{3}\right)_{3}$, $15 \mathrm{~mm}$ thiourea, and $1.2 \% \mathrm{HCl}$ was used to etch the dendrimer-printed areas.

\section{Acknowledgements}

The authors gratefully acknowledge support from the Nanotechnology network in the Netherlands 
Nanoned (Project No. TMM. 7125) and from the MESA+ Institute for Nanotechnology (SRO Nanofabrication). We are grateful to M. Smithers for SEM measurements and to our colleagues V. B. Sadhu and H. Xu for their help. Supporting Information is available online from Wiley InterScience or from the author.

Received: December 13, 2008

Revised: February 11, 2009

Published online: April 14, 2009

[1] L. Libioulle, A. Bietsch, H. Schmid, B. Michel, E. Delamarche, Langmuir 1999, 15, 300

[2] A. Kumar, G. M. Whitesides, Appl. Phys. Lett. 1993, 63, 2002.

[3] Y. N. Xia, G. M. Whitesides, Annu. Rev. Mater. Sci. 1998, 28, 153.

[4] Y. N. Xia, G. M. Whitesides, Langmuir 1997, 13, 2059.

[5] B. Michel, A. Bernard, A. Bietsch, E. Delamarche, M. Geissler, D. Juncker, H. Kind, J. P. Renault, H. Rothuizen, H. Schmid, P. Schmidt-Winkel, R. Stutz, H. Wolf, IBM J. Res. Dev. 2001, 45, 697.

[6] A. Bietsch, B. Michel, J. Appl. Phys. 2000, 88, 4310.

[7] K. G. Sharp, G. S. Blackman, N. J. Glassmaker, A. Jagota, C. Y. Hui, Langmuir 2004, 20,6430.

[8] Y. G. Y. Huang, W. X. Zhou, K. J. Hsia, E. Menard, J. U. Park, J. A. Rogers, A. G. Alleyne, Langmuir 2005, 21, 8058.

[9] H. Schmid, B. Michel, Macromolecules 2000, 33, 3042.

[10] D. Trimbach, K. Feldman, N. D. Spencer, D. J. Broer, C. W. M. Bastiaansen, Langmuir 2003, 19, 10957.

[11] G. Csucs, T. Kunzler, K. Feldman, F. Robin, N. D. Spencer, Langmuir 2003, 19, 6104 .
[12] E. Menard, L. Bilhaut, J. Zaumseil, J. A. Rogers, Langmuir 2004, 20, 6871.

[13] T. T. Truong, R. S. Lin, S. Jeon, H. H. Lee, J. Maria, A. Gaur, F. Hua, I. Meinel, J. A. Rogers, Langmuir 2007, 23, 2898.

[14] M. Tormen, T. Borzenko, B. Steffen, G. Schmidt, L. W. Molenkamp, Microelectron. Eng. 2002, 61-2, 469.

[15] T. W. Odom, J. C. Love, D. B. Wolfe, K. E. Paul, G. M. Whitesides, Langmuir 2002, 18, 5314.

[16] X. X. Duan, V. B. Sadhu, A. Perl, M. Péter, D. N. Reinhoudt, J. Huskens, Langmuir 2008, 24, 3621.

[17] E. Delamarche, C. Donzel, F. S. Kamounah, H. Wolf, M. Geissler, R. Stutz, P. Schmidt-Winkel, B. Michel, H. J. Mathieu, K. Schaumburg, Langmuir 2003, 19, 8749 .

[18] R. B. A. Sharpe, D. Burdinski, J. Huskens, H. J. W. Zandvliet, D. N. Reinhoudt, B. Poelsema, J. Am. Chem. Soc. 2005, 127, 10344

[19] X. Y. Lei, L. Wu, P. Deshpande, Z. N. Yu, W. Wu, H. X. Ge, S. Y. Chou, Nanotechnology 2003, 14, 786.

[20] M. J. Owen, P. J. Smith, J. Adhes. Sci. Technol. 1994, 8, 1063.

[21] G. S. Ferguson, M. K. Chaudhury, H. A. Biebuyck, G. M. Whitesides, Macromolecules 1993, 26, 5870.

[22] D. Y. Khang, H. Kang, T. Kim, H. H. Lee, Nano Lett. 2004, 4, 633.

[23] J. N. Lee, C. Park, G. M. Whitesides, Anal. Chem. 2003, 75, 6544.

[24] Y. N. Xia, X. M. Zhao, E. Kim, G. M. Whitesides, Chem. Mater. 1995, 7, 2332.

[25] Y. Zhao, E. Berenschot, H. Jansen, N. Tas, J. Huskens, M. Elwenspoek, Microelectron. Eng. in press.

[26] A. Perl, M. Péter, B. J. Ravoo, D. N. Reinhoudt, J. Huskens, Langmuir 2006, 22, 7568.

[27] M. K. Chaudhury, G. M. Whitesides, Science 1992, 256, 1539. 